\title{
$G$-SUPPLEMENTED LATTICES
}

\author{
HASAN HÜSEYİN ÖKTEN
}

Received 9 February, 2020

\begin{abstract}
In this work, g-supplemented lattices are defined and some properties of these lattices are investigated. g-small submodules and g-supplemented modules are generalized to lattices. Let $L$ be a lattice and $1=a_{1} \vee a_{2} \vee \ldots \vee a_{n}$ with $a_{i} \in L(1 \leq i \leq n)$. If $a_{i} / 0$ is g-supplemented for every $i=1,2, \ldots, n$, then $L$ is also g-supplemented. If $L$ is g-supplemented, then $1 / a$ is also g-supplemented for every $a \in L$. It is also defined the g-radical of a lattice $L$ and it is shown that if $L$ is g-supplemented, then $1 / r_{g}(L)$ is complemented.
\end{abstract}

2010 Mathematics Subject Classification: 06C05, 06C15

Keywords: Lattices, Essential Elements, Small Elements, Supplemented Lattices

\section{INTRODUCTION}

In this paper, every lattice is complete modular lattice with the smallest element 0 and the greatest element 1 . Let $L$ be a lattice, $x, y \in L$ and $x \leq y$. A sublattice $\{a \in L \mid x \leq a \leq y\}$ is called a quotient sublattice and denoted by $y / x$. An element $y$ of a lattice $L$ is called a complement of $x$ in $L$ if $x \wedge y=0$ and $x \vee y=1$, this case we denote $1=x \oplus y$ (in this case we call $x$ and $y$ are direct summands of $L$ ). $L$ is said to be complemented if each element has at least one complement in $L$. An element $x$ of $L$ is said to be small or superfluous and denoted by $x \ll L$ if $y=1$ for every $y \in L$ such that $x \vee y=1$. The meet of all maximal $(\neq 1)$ elements of a lattice $L$ is called the radical of $L$ and denoted by $r(L)$. An element $a$ of $L$ is called a supplement of $b$ in $L$ if it is minimal for $a \vee b=1 . a$ is a supplement of $b$ in a lattice $L$ if and only if $a \vee b=1$ and $a \wedge b \ll a / 0$. A lattice $L$ is called a supplemented lattice if every element of $L$ has a supplement in $L$. We say that an element $y$ of $L$ lies above an element $x$ of $L$ if $x \leq y$ and $y \ll 1 / x$. $L$ is said to be hollow if every element distinct from 1 is superfluous in $L$, and $L$ is said to be local if $L$ has the greatest element $(\neq 1)$. We say an element $x \in L$ has ample supplements in $L$ if for every $y \in L$ with $x \vee y=1, x$ has a supplement $z$ in $L$ with $z \leq y . L$ is said to be amply supplemented if every element of $L$ has ample supplements in $L$. It is clear that every amply supplemented lattice is supplemented. Let $L$ be a lattice and $k \in L$. If $t=0$ for very $t \in L$ with $k \wedge t=0$, then $k$ is called an essential element of $L$ and denoted by $k \unlhd L$. 
More informations about (amply) supplemented lattices are in [1, 2, 5, 9]. More results about (amply) supplemented modules are in $[8,12]$.

Definition 1. Let $L$ be a lattice and $a \in L$. If $b=1$ for every $b \unlhd L$ with $a \vee b=1$, then $a$ is called a generalized small (briefly, g-small) element of $L$ and denoted by $a \ll_{g} L$.

It is clear that every small element is g-small, but the converse is not true in general (See Example 1 and Example 2).

G-small elements generalize g-small submodules. G-small submodules are studied in $[6,7,11]$.

Lemma 1. Let $L$ be a lattice and $a, b, c, d \in L$. Then the followings are hold.

(i) If $a \leq b$ and $b \ll_{g} L$, then $a \ll_{g} L$.

(ii) If $a \ll_{g} b / 0$, then $a \ll_{g} t / 0$ for every $t \in L$ with $b \leq t$.

(iii) If $a \ll_{g} L$, then $a \vee b \ll_{g} 1 / b$.

(iv) If $a \ll_{g} b / 0$ and $c \ll_{g} d / 0$, then $a \vee c \ll_{g}(b \vee d) / 0$.

Proof. (i) Let $a \vee k=1$ with $k \unlhd L$. Since $a \leq b, b \vee k=1$ and since $b \ll_{g} L, k=1$. Hence $a \ll_{g} L$ as desired.

(ii) Let $t \in L$ with $b \leq t$ and let $a \vee k=t$ with $k \unlhd t / 0$. Here $k \wedge b \unlhd b / 0$. Since $a \leq b$, by modularity, $b=b \wedge t=b \wedge(a \vee k)=a \vee(k \wedge b)$ and since $a \ll_{g} b / 0, k \wedge b=b$ and $b \leq k$. Hence $a \leq k$ and $t=a \vee k=k$. Therefore, $a \ll_{g} t / 0$.

(iii) Let $a \vee b \vee k=1$ with $k \unlhd 1 / b$. Since $k \unlhd 1 / b$, we can easily see that $k \unlhd L$. Since $1=a \vee b \vee k=a \vee k$ and $a \ll_{g} L, k=1$. Hence $a \vee b \ll_{g} 1 / b$ as desired.

(iv) Let $a \vee c \vee k=b \vee d$ with $k \unlhd(b \vee d) / 0$. By $(i i) a \ll_{g}(b \vee d) / 0$ and $c \ll_{g}$ $(b \vee d) / 0$. Since $a \ll_{g}(b \vee d) / 0$ and $c \vee k \unlhd(b \vee d) / 0, c \vee k=b \vee d$ and since $c \ll g$ $(b \vee d) / 0, k=b \vee d$. Hence $a \vee c \ll_{g}(b \vee d) / 0$ as desired.

Corollary 1. If $a_{i} \ll_{g} b_{i} / 0$ for $a_{i}, b_{i} \in L(i=1,2, \ldots, n)$, then $a_{1} \vee a_{2} \vee \ldots \vee a_{n} \ll g$ $\left(b_{1} \vee b_{2} \vee \ldots \vee b_{n}\right) / 0$.

Proof. Clear from Lemma 1(iv).

Corollary 2. Let $a, b \in L$ and $a \leq b$. If $b \ll_{g} L$, then $b \ll_{g} 1 / a$.

Proof. Clear from Lemma 1(iii).

\section{G-SUPPLEMENTED LATTICES}

Definition 2. Let $L$ be a lattice and $a, b \in L$. If $1=a \vee b$ and $1=a \vee t$ with $t \unlhd b / 0$ implies that $t=b$, then $b$ is called a g-supplement of $a$ in $L$. If every element of $L$ has a g-supplement in $L$, then $L$ is called a g-supplemented lattice.

G-supplemented lattices generalize g-supplemented modules. G-supplemented modules are studied in [7]. Every supplemented lattice is g-supplemented. Hollow and local lattices are g-supplemented. 
Lemma 2. Let $L$ be a lattice and $a, b \in L$. Then $b$ is a g-supplement of $a$ in $L$ if and only if $1=a \vee b$ and $a \wedge b \ll_{g} b / 0$.

Proof. $(\Longrightarrow)$ Let $(a \wedge b) \vee k=b$ with $k \unlhd b / 0$. Then $1=a \vee b=a \vee(a \wedge b) \vee k=$ $a \vee k$ hold. Since $b$ is a g-supplement of $a$ in $L$ and $k \unlhd b / 0$, by definition, $k=b$. Hence $a \wedge b \ll_{g} b / 0$ as desired.

$(\Longleftarrow)$ Let $1=a \vee t$ with $t \unlhd b / 0$. Since $t \leq b$, by modularity, $b=b \wedge 1=b \wedge$ $(a \vee t)=(a \wedge b) \vee t$. Since $a \wedge b \ll_{g} b / 0, t=b$. Hence $b$ is a g-supplement of $a$ in $L$ as desired.

Lemma 3. Let $L$ be a lattice and $a, b \in L$. If $a \vee b$ has a g-supplement $x$ in $L$ and $(a \vee x) \wedge b$ has a g-supplement $y$ in $b / 0$, then $x \vee y$ is a g-supplement of a in $L$.

Proof. Since $x$ is a g-supplement of $a \vee b$ in $L$, by Lemma 2,

$$
1=a \vee b \vee x \text { and }(a \vee b) \wedge x \ll_{g} x / 0 .
$$

Since $y$ is a g-supplement of $(a \vee x) \wedge b$ in $b / 0$, by Lemma 2,

$$
b=((a \vee x) \wedge b) \vee y
$$

and

Then

$$
(a \vee x) \wedge y=(a \vee x) \wedge b \wedge y \ll_{g} y / 0
$$

$$
1=a \vee b \vee x=a \vee x \vee((a \vee x) \wedge b) \vee y=a \vee x \vee y
$$

and by Lemma 1 ,

$$
\begin{aligned}
a \wedge(x \vee y) & \leq((a \vee x) \wedge y) \vee((a \vee y) \wedge x) \\
& \leq((a \vee x) \wedge y) \vee((a \vee b) \wedge x) \ll_{g}(x \vee y) / 0 .
\end{aligned}
$$

Hence $x \vee y$ is a g-supplement of $a$ in $L$.

Corollary 3. Let $L$ be a lattice and $a, b \in L$. If $a \vee b$ has a g-supplement in $L$ and $b / 0$ is $g$-supplemented, then a has a g-supplement in $L$.

Proof. Clear from Lemma 3.

Lemma 4. Let $1=a \vee b$ with $a, b \in L$. If $a / 0$ and $b / 0$ are $g$-supplemented, then $L$ is also g-supplemented.

Proof. Let $x$ be any element of $L$. Then 0 is a g-supplement of $x \vee a \vee b$ in $L$ and since $b / 0$ is g-supplemented, by Corollary $3, x \vee a$ has a g-supplement in $L$. Since $a / 0$ is g-supplemented, again by Corollary $3, x$ has a g-supplement in $L$. Hence $L$ is g-supplemented.

Corollary 4. Let $1=a_{1} \vee a_{2} \vee \ldots \vee a_{n}$ with $a_{i} \in L(1 \leq i \leq n) . \quad$ If $a_{i} / 0$ is $g$ supplemented for every $i=1,2, \ldots, n$, then $L$ is also $g$-supplemented.

Proof. Clear from Lemma 4. 
Lemma 5. Let $L$ be a lattice and $a, b, c \in L$ with $c \leq a$. If $b$ is a g-supplement of $a$ in $L$, then $b \vee c$ is a g-supplement of a in $1 / c$.

Proof. Since $b$ is a g-supplement of $a$ in $L, 1=a \vee b$ and $a \wedge b \ll_{g} b / 0$. Since $a \wedge b \ll_{g} b / 0$, by Lemma 1 (ii),

$$
a \wedge b \ll_{g}(b \vee c) / 0
$$

and by Lemma 1 (iii),

$$
(a \wedge b) \vee c \ll_{g}(b \vee c) / c .
$$

Hence $1=a \vee b=a \vee b \vee c$ and $a \wedge(b \vee c)=(a \wedge b) \vee c \ll_{g}(b \vee c) / c$ and $b \vee c$ is a g-supplement of $a$ in $1 / c$.

Corollary 5. Let $L$ be a g-supplemented lattice. Then $1 / a$ is g-supplemented for every $a \in L$.

Proof. Clear from Lemma 5.

Definition 3. Let $L$ be a lattice and $t$ be a maximal $(\neq 1)$ element of $L$. If $t \unlhd L$, then $t$ is called a g-maximal element of $L$. The meet of all g-maximal elements of $L$ is called the g-radical of $L$ and denoted by $r_{g}(L)$. If $L$ have not any g-maximal elements, then we call $r_{g}(L)=1$.

Corollary 6. Let $L$ be a lattice. Then $r(L) \leq r_{g}(L)$.

Proof. Clear from definitions.

Lemma 6. Let $L$ be a lattice and $a \in L$. If $a \ll_{g} L$, then $a \leq r_{g}(L)$.

Proof. Assume $a \not \leq r_{g}(L)$. Then there exists a g-maximal element $t$ of $L$ with $a \not \leq t$. Since $t$ is maximal $(\neq 1)$ and $a \not \leq t, a \vee t=1$ and since $a \ll_{g} L$ and $t \unlhd L$, $t=1$. This is contradiction. Hence $a \leq r_{g}(L)$ as desired.

Lemma 7. Let $L$ be a lattice and $a \in L$. Then $r_{g}(a / 0) \leq r_{g}(L)$.

Proof. Let $t$ be any g-maximal element of $L$. If $a \leq t$, then $r_{g}(a / 0) \leq t$. If $a \not \leq t$, we can easily see that $a \wedge t$ is a g-maximal element of $a / 0$ and hence $r_{g}(a / 0) \leq t$. Therefore, $r_{g}(a / 0) \leq r_{g}(L)$.

Lemma 8. Let L be a g-supplemented lattice. Then $1 / r_{g}(L)$ is complemented.

Proof. Let $x$ be any element of $1 / r_{g}(L)$. Since $L$ is g-supplemented, $x$ has a gsupplement $y$ in $L$. Here $1=x \vee y$ and $x \wedge y \ll_{g} y / 0$. Since $x \wedge y \ll_{g} y / 0$, by Lemma 6 and Lemma 7, $x \wedge y \leq r_{g}(y / 0) \leq r_{g}(L)$. Hence $1=x \vee y \vee r_{g}(L)$ and

$$
x \wedge\left(y \vee r_{g}(L)\right)=(x \wedge y) \vee r_{g}(L)=r_{g}(L) .
$$

Therefore, $y \vee r_{g}(L)$ is a complement of $x$ in $1 / r_{g}(L)$ and $1 / r_{g}(L)$ is complemented. 
Let $x, y \in L$. It is defined a relation $\beta_{*}$ on the elements of $L$ by $x \beta_{*} y$ if and only if for every $t \in L$ with $1=x \vee t$ then $1=y \vee t$ and for every $k \in L$ with $1=y \vee k$ then $1=x \vee k$. (See [10, Definition 1]. More informations about $\beta_{*}$ relation are in [10]. More informations about $\beta^{*}$ relation on modules are in [4].

Corollary 7. Let $L$ be a g-supplemented lattice. Then $1 / r_{g}(L)$ is $\oplus$-supplemented.

Proof. Clear from [3, Definition 1] and Lemma 8.

Lemma 9. Let $L$ be a lattice and $a \beta_{*} b$ in $L$. If $a$ and $b$ have $g$-supplements in $L$, then they have the same g-supplements in $L$.

Proof. Let $x$ be a g-supplement of $a$ in $L$. Then $1=a \vee x$ and since $a \beta_{*} b$, we have $1=b \vee x$. Let $1=b \vee t$ with $t \unlhd x / 0$. Since $a \beta_{*} b$, we have $1=a \vee t$ and since $x$ is a g-supplement of $a$ in $L$, we have $t=x$. Hence $x$ is a g-supplement of $b$ in $L$. Similarly, interchanging the roles of $a$ and $b$ we can prove that each g-supplement of $b$ in $L$ is also a g-supplement of $a$ in $L$.

Corollary 8. Let $L$ be a lattice and a lies above $b$ in $L$. If $a$ and $b$ have $g$ supplements in $L$, then they have the same g-supplements in $L$.

Proof. By [10, Theorem 3], $a \beta_{*} b$ and by Lemma 9, the desired is obtained.

Lemma 10. Let $L$ be a lattice and $t \ll_{g} x / 0$ for every g-supplement element $x$ in $L$ and for every $t \ll_{g} L$ with $t \leq x$. If every element of $L$ is $\beta_{*}$ equivalent to a $g$-supplement element in $L$, then $L$ is $g$-supplemented.

Proof. Let $a \in L$. By hypothesis, there exists a g-supplement element $x$ in $L$ such that $a \beta_{*} x$. Let $x$ be a g-supplement of $b$ in $L$. By hypothesis, there exists a g-supplement element $y$ in $L$ with $b \beta_{*} y$. By Lemma 9, $x$ is a g-supplement of $y$ in $L$. Here $1=x \vee y$ and $x \wedge y \ll_{g} x / 0$. Since $x \wedge y \ll_{g} L$ and $y$ is a g-supplement element in $L$, by hypothesis, $x \wedge y \ll_{g} y / 0$. Then by Lemma 2, $y$ is a $g$-supplement of $x$ in $L$. Since $a \beta_{*} x$, by Lemma 9, $y$ is a $g$-supplement of $a$ in $L$. Hence $L$ is $g$-supplemented.

Corollary 9. Let $L$ be a lattice and $t \ll_{g} x / 0$ for every $g$-supplement element $x$ in $L$ and for every $t \ll_{g} L$ with $t \leq x$. If every element of $L$ lies above a $g$-supplement element in $L$, then $L$ is $g$-supplemented.

Proof. Clear from [10, Theorem 3] and Lemma 10.

Definition 4. Let $L$ be a lattice. If every element of $L$ with distinct from 1 is $\mathrm{g}$-small in $L$, then $L$ is called a g-hollow lattice.

Clearly we can see that every hollow lattice is g-hollow. But the converse is not true in general (See Example 2).

Proposition 1. Every g-hollow lattice is g-supplemented. 
Proof. Let $L$ be a g-hollow lattice. Then 1 is a g-supplement of every element of $L$ with distinct from 1 and 0 is a g-supplement of 1 in $L$. Hence $L$ is g-supplemented.

Proposition 2. Let $L$ be a lattice with $r_{g}(L) \neq 1$. The following conditions are equivalent.

(i) L is g-hollow.

(ii) $L$ is local.

(iii) L is hollow.

Proof. $(i) \Longrightarrow(i i)$ Let $x \in L$ and $x \neq 1$. Since $L$ is g-hollow, $x \ll_{g} L$ and by Lemma $6, x \leq r_{g}(L)$. By hypothesis, $r_{g}(L) \neq 1$. Hence $r_{g}(L)$ is the greatest element $(\neq 1)$ of $L$ and $L$ is local.

$($ ii $) \Longrightarrow($ iii $)$ and $(i i i) \Longrightarrow($ i) are clear.

Example 1. Let $L$ be a nonzero complemented lattice. Here $1 \ll_{g} L$, but not $1 \ll L$. 1 is a g-supplement of 1 in $L$, but 1 is not a supplement of 1 in $L$.

Example 2. Consider the lattice $L=\{0, a, b, 1\}$ given by the following diagram.

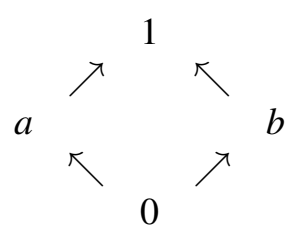

Then $L$ is g-hollow but not hollow. Here $1 \ll_{g} L$, but not $1 \ll L$. 1 is a g-supplement of 1 in $L$, but 1 is not a supplement of 1 in $L$. Here also $r(L)=0 \neq 1=r_{g}(L)$ hold.

Example 3. Consider the lattice $L=\{0, a, b, c, 1\}$ given by the following diagram.

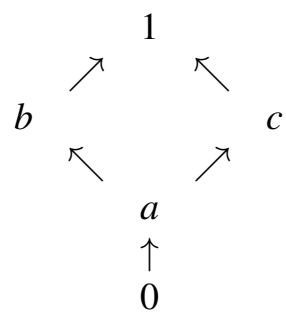

Then $L$ is g-supplemented but not g-hollow.

\section{REFERENCES}

[1] R. Alizade and S. E. Toksoy, "Cofinitely weak supplemented lattices," Indian J. Pure Appl. Math., vol. 40, no. 5, pp. 337-346, 2009.

[2] R. Alizade and S. E. Toksoy, "Cofinitely supplemented modular lattices," Arab. J. Sci. Eng., vol. 36, no. 6, pp. 919-923, 2011, doi: 10.1007/s13369-011-0095-Z. 
[3] Ç. Biçer and C. Nebiyev, “ $\oplus$-supplemented lattices," Miskolc Mathematical Notes, vol. 20, no. 2, pp. 773-780, 2019.

[4] G. F. Birkenmeier, F. Takil Mutlu, C. Nebiyev, N. Sokmez, and A. Tercan, "Goldie*-supplemented modules," Glasg. Math. J., vol. 52, no. A, pp. 41-52, 2010, doi: 10.1017/S0017089510000212.

[5] G. Călugăreanu, Lattice concepts of module theory, ser. Kluwer Texts in the Mathematical Sciences. Kluwer Academic Publishers, Dordrecht, 2000, vol. 22, doi: 10.1007/978-94-015-9588-9.

[6] B. Koşar, C. Nebiyev, and A. Pekin, "A generalization of g-supplemented modules," Miskolc Math. Notes, vol. 20, no. 1, pp. 345-352, 2019, doi: 10.18514/MMN.2019.2586.

[7] B. Koşar, C. Nebiyev, and N. Sökmez, "g-supplemented modules," Ukrainian Mathematical Journal, vol. 67, no. 6, pp. 861-864, 2015.

[8] C. Nebiyev and A. Pancar, "On supplement submodules," Ukrainian Math. J., vol. 65, no. 7, pp. 1071-1078, 2013, doi: 10.1007/s11253-013-0842-2.

[9] C. Nebiyev, "On supplement elements in lattices," Miskolc Math. Notes, vol. 20, no. 1, pp. 441449, 2019, doi: 10.18514/MMN.2019.2844.

[10] C. Nebiyev and H. H. Ökten, " $\beta_{*}$ relation on lattices," Miskolc Math. Notes, vol. 18, no. 2, pp. 993-999, 2017, doi: 10.18514/mmn.2017.1782.

[11] C. Nebiyev and H. H. Ökten, "Weakly G-supplemented modules," Eur. J. Pure Appl. Math., vol. 10, no. 3, pp. 521-528, 2017.

[12] R. Wisbauer, Foundations of module and ring theory, ser. Algebra, Logic and Applications. Gordon and Breach Science Publishers, Philadelphia, PA, 1991, vol. 3, a handbook for study and research.

\section{Author's address}

\section{Hasan Hüseyİn Ökten}

Amasya University, Technical Sciences Vocational School, Amasya, Turkey

E-mail address: hoktenegmail.com 